\title{
LA THEORISATION DE L'ACTIVITE POLITIQUE DES ONG EN RUSSIE
}

\author{
Vasilenko S.B., Ph.D. in Political Science in Paris-Dauphine University (PSL), Paris France \\ stepslav@yandex.ru
}

\begin{abstract}
Le conflit de l'État russe avec le champ associatifs'explique par la participation des ONG aux délibérations politiques dans les années 1990. La logique du contrôle sur les acteurs qui peuvent susciter les sentiments contestataires chez ses partisans conduit l'État centrale à restreindre l'activité des ONG qui "font la politique" par la loi sur les "agents étrangers". Alors, qu'est-ce que cela veut dire pour ces ONG de s'opposeraux autorités publiques et se construire en catégories politiques et civiques?
\end{abstract}

Keywords: ONG; société civile; agents étrangers; politique intérieur; Russie contemporaine

\section{ТЕОРЕТИЧЕСКИЙ АСПЕКТ ПОЛИТИЧЕСКОЙ ДЕЯТЕЛЬНОСТИ НЕПРАВИТЕЛЬСТВЕННЫХ ОРГАНИЗАЦИЙ В РОССИИ}

Василенко Степан Борисович, д-р полит. наук, Университет Париж-Дофин, Париж, Франция stepslav@yandex.ru

\begin{abstract}
Аннотация. Конфликт российского государства с организациями гражданского общества проистекает из участия НКО в политических процессах в 1990-х гг. Центральная власть, желая контролировать данных игроков, способных вызывать протестные настроения среди своей аудитории, законом об «иностранных агентах» ограничивает активность НКО, «занимающихся политикой». Что в таком случае для этих НКО значит быть в оппозиции к власти и позиционировать себя в политических и гражданских категориях?

Ключевые слова: НКО; гражданское общество; иностранные агенты; внутренняя политика; современная Россия
\end{abstract}

\section{L'ACTUALITÉ POLITIQUE: LES ENJEUX AUTOUR DE NOMINATION}

Le 29 juin 2012, la Chambre basse du Parlement de Russie a commencé l'examen d'une nouvelle loi qui visait à munir certaines ONG russes qui s'occupaient des questions politiques d'un statut d'«agent étranger» (en russe cette expression renvoie à l'idée d'un espionnage). Ce statut symbolique devait toucher des ONG qui sont financées, même partiellement, par des États étrangers, par des organisations internationales ou par des citoyens étrangers. L'ambiguiité de la situation autour de cette loi consiste dans le fait que l'activité politique est une notion qui n'est pas bien définie. Ainsi, cela permettrait d'imposer à certaines ONG le statut d' «agent étranger»en fonction de critères souples. Dans le contexte des manifestations et des mouvements pour les «élections libres» (printemps
2012) soutenues par des plusieurs ONG russes, cette tentative est perçue par des acteurs de ce champ associatif comme un prolongement de la politique de restriction qui a pour but d'empêcher toute activité des organisations non gouvernementales. L'enjeu de cette loi est de délégitimer ces acteurs en tant que «mains gauches» des États étrangers. Dans son article publié le 27 février 2012 lors de la campagne présidentielle, le président russe Vladimir Poutine explique la politique que mènerait son cabinet envers les ONG:

«Il faut faire attention à ne pas confondre la liberté d'expression et l'activité politique normale avec l'utilisation illégale des outils de soft power [...] Nous ne pouvons accepter l'activité de pseudos ONG et d'autres structures qui, fortes des appuis étrangers dont elles bénéficient, visent à la déstabilisation d'un pays...» (http://mn.ru/politics/20120227/312306749.html). 
Les ONG actives les plus connues (Golos, Memorial, Human Rights Watch, Amnesty International, groupe Helsinki de Moscou) qui luttent pour les droits de l'homme sont les premières destinataires de cette loi, car elles ne cachent pas le fait que leurs financements vient partiellement ou totalement de l'étranger, d'après les données qu'elles transmettent au ministère de la Justice. Pourtant, les portes-paroles de certaines spécialisées dans la défense des droits de l'homme déclarent qu'ils ne porteront jamais le titre des «agents étrangers» et que leur activité va continuer.

Pourtant, cela n'a pas empêché le président russe Vladimir Poutine de signer le projet de loi du 20 juillet 2012. Désormais, toute ONG qui travaille sur la politique et bénéficie d'une aide financière provenant de l'étranger doit se faire enregistrer et s'afficher comme «agent étranger». La loi Fédérale $\mathrm{N}-121 \mathrm{FZ}$ datée de 20 juillet 2012 définit également des obligations fiscales plus strictes que celles qui sont appliquées à d'autres $\mathrm{ONG}^{1}$. Le refus de se déclarer comme «agent étranger» a été assimilé à une infraction de la loi avec des peines d'amende de cent mille roubles à trois cent mille roubles pour une personne physique et de trois cent mille roubles à cinq cent mille roubles pour une personne morale $^{2}$. En outre, la loi Fédérale n’a pas prévu la procédure qui permettrait de supprimer le statut d' «agent étranger». Le ministère de la Justice a également obtenu le pouvoir d'imposer ce statut à toute ONG dont l'activité correspond aux critères évoqués dans la lois Fédérale.

\section{LE REGARD RÉTROSPECTIF SUR LES RELATIONS ENTRE LES ONG ET ÉTAT RUSSE}

Le conflit de l'État russe avec le champ associatif international ne peut être compris que dans son historicité. Il faut comprendre l'émergence soudaine de ce champ des défenseurs des droits de l'homme dans les années 1990. Contrairement à la neutralité politique présumée qui caracté-

\footnotetext{
${ }^{1}$ Ainsi, à chaque trimestre des ONG-«agents étrangers» doivent informer le Ministère de la Justice non seulement sur les sommes d'argents et des biens matériels accordés par des sources étrangères, mais aussi sur les buts des dépenses et des frais réels.

${ }^{2} \mathrm{Si}$ l'on recalcule ces amendes au taux de change de juillet 2012, il s'agit de la somme de 2500 à 7500 euro pour une personne physique et de 7500 à 12500 euro pour une personne morale, soit 40 rouble pour 1 euro. Pour donner le sens à ces chiffres, il est indispensable de dire que la moyenne des salaires en Russie en juillet 2012 était égale à 26683 rouble, soit 667 euro.
}

rise les ONG de défense des droits de l'homme en Occident, la participation à des délibérations politiques fait partie de l' habitus des associations russes dès la chute de l'URSS. On constate que dans l'histoire russe récente, un engagement civique s'entremêle avec le militantisme politique. De ce fait, les défenseurs des droits de l'homme se perçoivent comme des acteurs légitimes du jeu politique. Cette tradition qui date des années 1990 est très présente chez des personnes ayant des profils dissidents et intellectuels [1]. De la même manière, dans le contexte autoritaire de la Russie de 2012, l'État se sent également légitime à exclure progressivement ces groupes des contours politiques lorsqu'ils entrent en concurrence avec le pouvoir ou alimentent les sentiments contestataires chez ses partisans.

Cela pose quelques questions, notamment de savoir dans quelle mesure l'apparition des structures internationales comme des ONG en droits de l'homme a été initiée par le gouvernement russe. Peut-on inscrire la mise en vigueur des ONG au même titre que le transfert des politiques publiques? Notre hypothèse est qu'après la dissolution de l'URSS, la réforme de l'État russe impliquait la nécessité de trouver des nouveaux moyens d'interaction avec la population. Les ONG de défense des droits de l'homme permettraient à l'État de rejeter la création «d'une société civile» (une notion qu'on met sur la critique) sur le dos de ces acteurs internationaux jusqu'au moment où les ONG sont entrées en concurrence avec l'État dans le champ politique. Une autre explication est aussi possible si l'on admet que l'implantation des ONG est une importation des technologies de l'extérieur. Dans ce cas-là, la confrontation avec l'État russe était inévitable et, aux yeux des pouvoirs russes, les ONG devaient légitimer leur présence en Russie. Ces questions se posent à partir de l'accès au pouvoir de Vladimir Poutine.

La prédominance de la logique du contrôle de l'État sur les ONG russes plonge ses racines au début des années 2000. L’idée de départ était de diminuer l'influence des États étrangers sur la politique russe, car les ONG étaient considérées comme des «acteurs agissant au nom d'une gouvernance globale dont elles sont l'un des outils» [2], c'est également ce que montre Bernard Hours au sujet de l'Ouzbekistan. C'est la même logique qui alimente les contraintes de plus en plus imposantes sur les ONG pour la défense des droits de l'homme en Russie (les enquêtes policières sur 
les sources de financement des ONG lancées en 2010, p.ex.). Dans son ouvrage Les ONG en Russie post-soviétique (2011), la chercheuse canadienne Agnès Blais montre à partir de l'exemple des associations caritatives comment évolue la politique de l'État en Russie envers des ONG et comment le champ associatif se construit de nouveau (en URSS il n'existait pas d'ONG) et se développe progressivement [3]. Dans cet article, on prolonge ces pistes en mettant l'accent sur la trajectoire collective des ONG de défense des droits de l'homme. Il s'agit de voir comment les acteurs inventent ce qui est ONG dans l'espace public russe et comment ces ONG deviennent une catégorie politique. Comment ces ONG définissent-elles leurs limites et leurs rapport à la politique? Comment investissent-elle, à leur manière, la vie politique?

Dans les conditions russes où l'accès à la participation politique est bloquée ou limitée, est-il possible de faire la politique autrement? Dans les années après 2003 le champ associatif russe répond de deux manières. D'une part, par la recomposition des alliances avec des mouvements d'oppositions des défenseurs des droits de l'homme éminents se trouvent à côté des militants radicaux dans la rue, qui devient l'un des espaces exclusifs pour s'exprimer ouvertement [4]. D'autre part, l'inertie politique mène à l'auto-limitation des ONG de défense des droits de l'homme qui refusent de s'opposer à l'État sous forme de contestations dans la rue. Pour rester en politique, sans entrer en confrontation directe avec les autorités russes, les ONG recourent à l'usage militant et politique du droit. C'est pourquoi on voit des anciens députés (Yabloko, SPS) s'investir dans des ONG de défense des droits de l'homme et convertir les expertises juridiques accordées aux citoyens dans le capital politique [5].

Dans un rapport de 2008 intitulé «Bureaucratie oppressante» (http://www.hrw.org/reports/2008/02/19/choking-bureaucracy), Human Rights Watch décrit les relations de l'État russe avec des ONG ainsi que les blocages auxquels elles se heurtent dans leur activité, à savoir la création d'une «société civile» en Russie. Par contre, comme le décrit bien Michel Camau: «Polysémique, la notion de société civile tend à structurer le champ de confrontation de stratégies discursives, où se rejoignent et s'opposent l'autoritarisme et ses adversaires, tenants de l'État et acteurs "non gouvernementaux"» [6]. On postule que les ONG de défense des droits de l'homme tendent à penser la société russe dans le cadre de «la téléologie de la démocratisation» [7, p. 9] qui définit leurs relations avec l'Etat et la population russes. En agissant dans ce paradigme de pensée, les ONG en droits de l'homme n'ont d'autre choix que d'entrer en conflit avec l'État. Comme le concept de «société civile» est théorique et éphémère, l'État ne pourra jamais répondre aux attentes des ONG en droits de l'homme.

\section{S'IMPOSER À L'ETAT EN RUSSIE ET AILLEURS}

De la même manière, les ONG en droits de l'homme ne trouvent pas de soutien dans l'opinion publique, qui varie de l'indifférence à l'hostilité envers des ONG dont les buts et les résultats ne sont pas bien visibles. Ce paradoxe est bien saisi par des politistes français du même ouvrage: «En deux mots: le démocratisme a tué l'horizon d'attente démocratique qui se trouve aujourd'hui totalement décrédibilisé auprès des populations et des dirigeants, porté à bout de bras par quelques ONG locales grassement financées par les institutions internationales» [7, p. 14]. Étant enfermées dans une logique développementaliste, les ONG en droits de l'homme cherchent les marges de manœuvre pour légitimer leur présence. Il en résulte que le discours sur les droits de l'homme se transforme non seulement dans le discours politique (société civile, la démocratie), mais aussi dans l'engagement militant - (dans les années 2010 chaque mois Ludmila Alexeeva - leader groupe de Helsinki - organisait des rallyes avec le leader du parti national-bolchevik Edouard Limonov sous l'égide du mouvement politique «Autre Russie»).

Dans les conditions où l'État russe tend à rétrécir l'espace des «mouvement sociaux éphémères» [8], comment se fait-il que les ONG en droits de l'homme continuent à rester les acteurs du débat public? Comment se fait-il que ces ONG se constituent graduellement en tant qu' acteurs qui entrent dans des rapports de force avec l'État? Dans notre démarche il nous semble nécessaire d'éviter de succomber au discours de victimisation tenu par des responsables des ONG et par des journalistes. Il nous semble que les ONG restent des acteurs puissants qui ont suffisamment de marge pour contourner les limitations des libertés imposées par l'État. Les liens entre médias et organisations internationales leur permettent de jouer un rôle non-négligeable dans l'opinion publique pour légitimer leur existence. Dans une situation de conflit, la mobilisation des ressources par des acteurs associatifs peut s'effectuer au niveau national 
aussi bien qu'au au niveau international comme l'expliquent Elena Aoun et Joël Ficet: «Ces réseaux (dénommés coalitions, ligues, alliances...) rassemblent des organisations partageant des valeurs proches et des préoccupations similaires, et tendent à la mise en commun des ressources de chacun (informations, moyen logistiques) dans la perspective de peser sur les circuits institutionnels de la décision politique» [9]. Il nous semble que l'externalisation des conflits internes est la manière de s'imposer à l'État russe. Dans ce contexte il est possible d'évoquer l'exemple d'un pays «en transition démocratique», celui de la Turquie, où depuis de la fin des années 1990 la confédération syndicale de la fonction publique (KESK) mobilise non seulement ses adhérents mais s'assure aussi du soutien de la $\mathrm{CES}^{3}$ pour faire face aux initiatives «autoritaires» du gouvernement turc de limiter les droits syndicaux. En examinant les mobilisations de 1998 et 2001 Emre Öngün montre que le gouvernement essaye de maintenir l'équilibre entre sa volonté d'adhérer à l'Union Européenne et de sécuriser en même temps son pouvoir. En 1998 l'intervention des acteurs internationaux force l'État turc à reculer mais, en 2001, le gouvernement arrive à imposer la loi sur le syndicalisme sans prendre compte les appels du CES [10]. Cette comparaison est d'autant plus intéressante compte tenu de la simultanéité des processus en Russie et en Turquie. Ainsi, à la fin année 1990 le gouvernement russe commence ses premières tentatives de réduire le rôle des associations dans l'espace politique russe ayant partiellement accompli ce but en 2001 [11].

\section{LES ONG COMME DES GROUPES D'INTÉRÊT}

Pourtant la mobilisation des ressources internationales nous permet de s'interroger sur les pratiques et les populations défendues par des ONG en droits de l'homme. Nous allons analyser des «dilemmes pratiques et interactions stratégiques en explorant la manière dont les individus investissent avec leur savoir-être et leur savoir-faire, leur motif et leurs perceptions» [12]. Cela signifie de s'intéresser au niveau local qui se définit par des pratiques quotidiennes et par l'action concrète en faveur des populations ciblées. Il s'agit de se débarrasser de l'opinion sur l'activité de certaines ONG sensibilisées aux droits de l'homme. Les structures les plus médiatisées comme Memorial, Groupe Helsinki

\footnotetext{
${ }^{3}$ Confédération Européene des Syndycats qui défend intérêts des travailleurs auprès des institutions de l'Union européenne.
}

de Moscou sont souvent accusées de n'aider que des riches (notamment un oligarque emprisonné Khodorkovsky), des journalistes et des Tchétchènes au détriment des «vraies victimes» du régime. Il est possible de voir comment s'entremêlent les conflits ethniques, de classe et d'intérêts qui entourent ce type d'organisation. Alors que les ONG en défense des droits de l'homme ne représentent pas toutes les citoyens, comment ces «groupes d'intérêt» [13] gagnent-ils la légitimité de parler au nom de tous? Est-ce que leurs actions et leurs pratiques quotidiennes permettent-elles de revendiquer une positions d'«entrepreneurs de morale» [14]? Cette critique est souvent formulée par différents acteurs (des groupes des nationalistes russes, des associations des droits des étrangers, des médias russes).

\section{L'EXEMPLE DE LA RUSSIE POUR D'AUTRES PAYS}

Notre article voulait répondre à la question de savoir quel était l'enjeu politique d'adopter des lois sur des ONG-«agents étrangères» en 2012. Notre réponse est que, pour l'Etat russe, ces lois permettent non seulement de réduire encore plus le pluralisme politique mais aussi de contrôler des «formes» de la société civile à l'occidentale. C'est pourquoi des explications officielles soulignent le fait que certaines ONG «font la politique» alors qu'elles sont financées par des tiers Etats. Par contre, nos observations sur les liens entre l'engagement politique chez les ONG et le rétrécissement d'une marge de manœuvre pour un champ associatif comme une pratique politique de l'Etat peuvent s'inscrire dans un contexte plus globale que celui de la Russie postsoviétique. Ainsi, la politique restrictive vis-à-vis les ONG de défense des droits de l'homme n'est plus un phénomène propre à la Russie. Depuis 2013 des pays de l'ex-USSR (comme Kazakhstan, l'Arménie, la Kirghizie, le Tadjikistan et l'Azerbaïdjan) et des pays en développement comme Bosnie, l'Egypte, le Venezuela et bien d'autres reprennent les mêmes arguments en adoptant ${ }^{4}$ des lois similaires à celles des «agents étrangers» en Russie. Il s'agit également d'un contrôle plus strict sur les sources de financements des ONG et leur participation dans la politique publique. Toutes ces lois renforcent le pouvoir central et affaiblissent le champ associatif de ces pays.

\footnotetext{
${ }^{4}$ Un rapport intitulé «Russia's bad exemple» sur ce sujet est publié par une ONG de défense des droits de l'homme américain «Human rights first» en février 2016.
} 


\section{REFERENCES}

1. Sigman C. Les mutations de l'espace politique en Russie pendant la perestrojka (1986-1991): Les clubs politiques informels de Moscou et leurs dirigeants [The transformations of political space in Russia during perestroika,1986-1991: Informal political clubs in Moscow and their leaders], thèse de doctorat en science politique soutenue à l'Université Paris I: Panthéon-Sorbonne; 2007.

2. Hours B. Les ONG au service de la gouvernance globale: le cas de l'Ouzbékistan [The NGOs serving global governance: the case of Uzbekistan]. Autrepart. 2005;3(35):115-126.

3. Blais A. Les ONG en Russie post-soviétique [The NGOs in post-soviet Russia]. Presses de l'Université Laval; 2011.

4. Vaissié C. Étouffement et renaissance des oppositions en Russie (2000-2010) [The crushing and the revival of the oppositions in Russia, 2000-2010]. Hérodote, 138;3(138):109-126.

5. Richard H. S'opposer par le droit: vulgarisation et usages politiques du droit de la copropriété en Russie postcommuniste [Opposition through law: the popularization and political uses of the law of co-ownership in post-communist Russia]. Critique internationale. 2012;2(55):35-50.

6. Camau M. Sociétés civiles «réelles» et téléologie de la démocratisation ["Real" civil societies and the theology of democratisation]. Revue internationale de politique comparée. 2002;2(9):213-232.

7. Dabène O., Geisser V. et Masardier G. Autoritarismes démocratiques et démocraties autoritaires au XXe siècle. Convergence Nord/Sud [The democratic authoritarianism and authoritarian democracies in XXth century. North/South convergence]. Paris: La Découverte; 2008.

8. Agrikoliansky É. La ligue française des droits de l'homme et du citoyen depuis 1945. Sociologie d'un engagement civique [The ligue française des droits de l'homme et du citoyen since 1945. Sociology of a civic engagement]. Paris: L'Harmattan; 2002.

9. Aoun E., Ficet J. La mobilisation d'un réseau d'ONG. La coalition française pour la Cour pénale internationale et la ratification du statut de Rome par la France [Mobilization of a network of a NGO. The French coalition for the International Criminal Court and the ratification of the Rome statute by France]. Siméant J. et Dauvin P.O.N.G. et humanitaire [NGO and aid work]. Paris: L'Harmattan; 2004.

10. Öngün E. Efficacité et recours aux protecteurs étrangers en contexte autoritaire [The efficiency and the use of foreign protectors in authoritarian context]. Dabène O., Geisser V. et Masardier G. Autoritarismes démocratiques et démocraties autoritaires au XXe siècle. Convergence Nord/Sud [The democratic authoritarianism and authoritarian democracies in XXth century. North/South convergence]. Paris: La Découverte; 2008.

11. Daucé F. Associations et partis en Russie: les (en) jeux de la différenciation [Associations and parties in Russia: what's at stake in the game of differentiation]. Critique internationale. 2012;2(55):17-34.

12. Fillieule O. Tombeau pour Charles Tilly. Répertoires, perfomences et stratégie d'action [A tomb for Charles Tilly. Repertoires, performances and action strategies]. In Penser les mouvement sociaux [To think about social movements] sous la dir de Fillieule O., Agrikoliansky É., Sommier I. Paris: La Découverte; 2010.

13. Offerlé M. Sociologie des groupes d'intérêt [The sociology of interest groups]. Paris, Montchrestien; 1994.

14. Becker H. Outsiders. Études de sociologie de la déviance [Outsiders. Studies in the sociology of deviance] Paris: A.-M. Métailié; 1985. 